\title{
AS EMPRESAS BRASILEIRAS DE CAPITAL FECHADO SÃO MAIS AGRESSIVAS EM SUAS EFFECTIVE TAX RATE (ETR) DO QUE AS DE CAPITAL ABERTO?
}

\section{LAS EMPRESAS BRASILEÑAS DE CAPITAL CERRADO SON MÁS AGRESIVAS EN SU EFFECTIVO TAX RATE (ETR) QUE LAS DE CAPITAL ABIERTO?}

\author{
BRAZILIAN CLOSED CAPITAL COMPANIES ARE MORE AGGRESSIVE IN THEIR \\ EFFECTIVE TAX RATE (ETR) THAN OPEN CAPITAL?
}

\footnotetext{
Robério Dantas de França, Programa de Pós-Graduação em Ciências Contábeis (UFPB), João Pessoa, PB, Brasil

Paulo Aguiar Monte, Programa de PósGraduação em Ciências Contábeis (UFPB), João Pessoa, PB, Brasil

Artigo Submetido em 18.12.2017. Aprovado em 16.02.2018.

Avaliado pelo processo de double blind review

e-mail para contato: roberiodantas@terra.co $\underline{\mathrm{m} . b r}$
}

\section{RESUMO}

No Brasil existe uma lacuna de estudos comparativos sobre a determinação da Effective Tax Rate (ETR) entre as empresas abertas e fechadas. Neste contexto, este trabalho busca contribuir para a incipiente discussão assumindo a hipótese de que as empresas brasileiras de capital fechado são propensas a apresentarem ETR mais agressivas do que as de capital aberto. Para isso, foram estimados modelos de regressão (OLS - Ordinary Least Square, e LSDV - Least Square Dummy Variables) para investigar o poder explicativo da variável de interesse (empresas de capital fechado ou aberto) em relação a variável dependente (ETR), controlando por setores econômicos e ciclo de vida organizacional de 668 firmas brasileiras no período de 2010 a 2015. Os resultados indicaram que: (i) empresas brasileiras de capital fechado apresentam ETRs menores em relação às empresas abertas (aproximadamente $45,53 \%)$ e, (ii) os estágios de maturidade e turbulência do ciclo de vida organizacional têm relação indireta com a ETR (aproximadamente 6\%).

Palavras-chave: Empresasde Capital Fechado e Aberto.Effective Tax Rate. Setores Econômicos. Ciclo de Vida Organizacional.

\begin{abstract}
In Brazil, there is a gap of comparative studies on the determination of the Effective Tax Rate (ETR) between privately and publicly held company. In this context, this paper seeks to contribute to the incipient discussion assuming the hypothesis that Brazilian privately held companies are likely to assume more aggressive ETRs than publicly ones. For this, regression models (OLS - Ordinary Least Square, and LSDV Least Square Dummy Variables) were estimated to investigate the explanatory power of the main variable (public or privately held company) on the dependent variable (ETR), controlling forindustry sectors and organizational life cycle (control variables) of 668 Brazilian firms from 2010 to 2015. The results indicate that: (i) Brazilian privately held companies have lower ETRs compared to public firms (around 45.53\%), and (ii) the maturity and turbulence stages of the organizational life cycle have an indirectrelation with ETR (around 6\%).
\end{abstract}

Key-words: Privately Held Company and Publicly Held Company. Effective Tax Rate. Industry Sectors. Organizational Life Cycle. 


\section{(9REUNIR}

RESUMEN

En Brasil hay un vacio de estudios comparativos acerca de la determinación Effective Tax Rate (ETR) entre las empresas abiertas y cerradas. En este contexto, este trabajo busca contribuir para nuevo debate asumiendo la hipótesis de que las empresas brasileñas de capital cerrado son propensas a presentar ETR más agresivas que las de capital abierto. Para eso, estimamos modelos de regresión (OLS - OrdinaryLeast Square, e LSDV - Least Square Dummy Variables) para investigar el poder explicativo de la variable de interés (empresas de capital cerrado o abierto) en relación a la variable dependiente (ETR), controlando por sectores económicos y ciclo de vida organizacional de 668 empresas brasileñas en el período de 2010 a 2015. Los resultados indicaron que: (i) empresas brasileñas de capital cerrado presentan ETRs menores en relación a las empresas abiertas (aproximadamente 45,53\%) e, (ii) las etapas de madurez y turbulencia del ciclo de vida organizacional tienen relación indirecta con la ETR (aproximadamente el 6\%).

Palabras clave: Empresas de Capital Cerrado y Abierto. Effective Tax Rate. Sectores Económicos. Ciclo de Vida Organizacional.

\section{INTRODUÇÃO}

A investigação sobre os impactos das características individuais das empresas sobre a Effective Tax Rate - ETR têm estimulado estudos na área de contabilidade e tributos(GUPTA;NEWBERRY, 1997, DESAI; DHARMAPALA, 2006, DYRENG;HANLON; MAYDEW, 2008, ARMSTRONG;BLOUIN; LARCKER, 2012;SANTOS;CAVALCANTE; RODRIGUES, 2013;KRAFT, 2014; PARISI, 2016; HASAN et. al., 2016). Entretanto, poucos têm foco no contexto das empresas de capital fechado ou no que diz respeito às diferenças entre essas empresas e as de capital aberto.

A Effective Tax Rate (ETR) é frequentemente utilizada nas pesquisas como proxy para identificar atividades de tax avoidance, que por sua vez representa reduções dos impostos sobre o lucro, não distinguindo sua legalidade e incorporando todas as transações e arranjos que poderiam resultar em uma diminuição no valor do imposto sobre o lucro (SHACKELFORD; SHEVLIN, 2001; REGO, 2003; HANLON; HEITZMAN, 2010). Em geral, as empresas apresentam ETRs abaixo da taxa nominal, sugerindo que a empresa estaria envolvida em atividades de tax avoidance.

Diferentes níveis de tax avoidance podem estar relacionados ao fato da empresa ter o seu capital aberto ou fechado, sendo um dos motivos a quantidade de informações financeiras que as empresas abertas estão sujeitas a divulgar. Com isso, estratégias tributárias agressivas seriam identificadas com mais facilidade pelas autoridades fiscais e os custos associados com essas estratégias mais elevados (HOPE; MA; THOMAS, 2013; JACOB; ROHLFING-BASTIAN; SANDNER, 2014). Por sua vez, a pesquisa anterior sugere que as empresas de capital fechado sofrem menor pressão do mercado e têm custos mais baixos quando divulgam lucros menores. Dessa forma, essas empresas tenderiam a se envolver em estratégias tributárias agressivas por 
se tornar menos oneroso reduzir os lucros contábeis e tributários (CLOYD; PRATT; STOCK, 1996; MILLS; NEWBERRY, 2001; LIN; MILLS; ZHANG, 2013).

As empresas teriam outras razões para se envolver em estratégias de tax avoidance, por exemplo, quando os objetivos de gestão se baseiam em medidas de lucro após os impostos, caso em que os gerentes seriam incentivados a alcançarem suas metas através dessas estratégias (DESAl; DHARMAPALA, 2006). Além desses motivos, a reputação corporativa, a assimetria informacional e os incentivos gerenciais dos executivos (DYRENG; HANLON; MAYDEW, 2010; GRAHAM et. al., 2014; DYRENG et. al., 2017) também podem ocasionar diferenças entre as ETRs de empresas abertas e fechadas.

Com base nessecontexto e considerando a escassez de pesquisas sobre o tema no Brasil, têm-se como objetivoinvestigar se as empresas brasileiras de capital fechado são propensas a apresentarem Effective Tax Rate (ETR) mais agressivas do que as de capital aberto. E, de forma secundária, avaliar a influência dos setores econômicos, o ciclo de vida organizacional e outros determinantes da ETR (tamanho, rentabilidade, alavancagem e intensidade do capital e do estoque) numa amostra de 668 empresas brasileiras.

\section{REVISÃO DA LITERATURA E HIPÓTESE DE PESQUISA}

Esta sessão descreve uma breve revisão da literatura relacionada ao tema, bem como apresenta a hipótese de pesquisa.

\subsection{Effective Tax Rate e as empresas de capital aberto e fechado}

As pesquisas sobre tax avoidance que investigam os determinantes da Effective Tax Rate (ETR) com foco no contexto das empresas de capital fechado ou no que diz respeito às diferenças entre essas empresas e as de capital aberto são escassas (CLOYD; PRATT; STOCK, 1996; DHALIWAL; GLEASON; MILLS, 2004; HOPE; MA; THOMAS, 2013; LIN; MILLS; ZHANG, 2013; JACOB; ROHLFING-BASTIAN; SANDNER, 2014, PIERK, 2016). No Brasil, não foram encontradas pesquisas que analisam essa associação com a ETR de forma direta, sendo, portanto, uma contribuição inédita a ser explorada.

As empresas abertas sofrem maior pressão do mercado, levando-as a considerar mais cuidadosamente a importância da informação financeira e a qualidade dos lucros divulgados (CLOYD; PRATT; STOCK, 1996; MILLS; SANSING, 2000; JACOB; ROHLFING-BASTIAN; SANDNER, 2014; PIERK, 2016). Cloyd, Pratt e Stock (1996) argumentam que as empresas de capital aberto incorrem em custos não tributários maiores quando relatam lucros mais baixos. Dessa forma, para um determinado nível esperado de economia de impostos essas empresas estarão menos inclinadas a se envolver com estratégias de tax avoidance. Para os autores, as firmas de capital aberto são menos propensas à conformidade do que as de capital fechado com a justificativa de que elas seriam mais sensíveis aos custos de divulgação financeira, conclusão também compartilhada por Penno e Simon (1986) e Mills e Newberry (2001).

Por sua vez, devido a um menor nível de divulgação financeira, as empresas de capital fechado teriam incentivos ao gerenciamento tributário (LIN; MILLS; ZHANG, 2013). Vale salientar que no Brasil, a Lei 11.638/07, determina às sociedades de grande porte que se 
apliquem os mesmos efeitos da Lei 6.404/76. Desse modo, as empresas de capital fechado consideradas de grande porte estão sujeitas as mesmas exigências de divulgação das empresas de capital aberto. Ainda assim, as empresas de capital aberto sofrem pressão adicional em sua divulgação devido as características de sua propriedade e controle, maior atenção das autoridades tributárias, cobertura de analistas de mercado e influência de investidores sofisticados, além de custos de agência mais elevados e estruturas mais complexas de governança.

\subsection{Outros determinantes da Effective Tax Rate}

O setor econômico é um determinante importante para os estudos da área tributária, uma vez que determinados setores apresentam regimes específicos de tributação e outros são alvo de incentivos fiscais. Lopes (2012) constatou que o tamanho da firma é negativamente associado à ETR, mas seus resultados indicam que determinados setores, como o financeiro, apresentam ETRs menores em relação aos de construção civil e têxtil. Por sua vez, Santos, Cavalcante e Rodrigues (2013) demonstram que no setor de energia elétrica as variáveis que representam otamanho, a lucratividade e a intensidade do estoque explicam as variações da ETR.

A inclusão dos Estágios do Ciclo de Vida organizacional(ECV) na análise sobre os determinantes da ETR no Brasil é pouco explorada. Hasanet. al., (2016) mostram que o ciclo de vida organizacional é um determinante importante do tax avoidance (estratégias para diminuição dos impostos sobre o lucro). No Brasil, Silva (2016) constatou que os ECVs iniciais e avançados possuem maiores níveis de planejamento tributário, numa amostra que contemplou as empresas de capital aberto do índice IBrX 100 da BM\&FBovespa.

Em relação ao tamanho da firma, a discussão clássica sobre a associação com a ETR se baseia em duas visões concorrentes: a teoria do custo político (ZIMMERMAN, 1983; WATTS;ZIMMERMAN, 1986) e a teoria do poder político (SIEGFRIED, 1972). Sob a ótica do custo político, a visibilidade das empresas maiores e mais prósperas as tornam vítimas de ações reguladoras e transferência de riqueza por parte do governo. A teoria do poder político, por sua vez, defende que as grandes empresas teriam ETRs mais baixas porque possuem recursos substanciais para manipular o processo político ao seu favor. Além disso, as grandes empresas se tornariam especializadas em planejamento tributário por possuírem conhecimento técnico superior e por consequência, usariam essa vantagem para otimizar suas economias de impostos.

As pesquisas que buscam uma relação entre a ETR e o tamanho da firma evoluíram, mas os resultados ainda são contraditórios (REGO, 2003; DYRENG et. al., 2008; KRAFT, 2014). No Brasil, pode-se destacar o trabalho de Santoset. al.,(2013) que investigou se a ETR das empresas de capital aberto poderia ser explicada pelo tamanho da firma. Os resultados mostraram uma relação positiva do tamanho com a tributação, sendo consistente com a hipótese dos custos políticos. No entanto, observou-se também uma relação não linear, sugerindo que a partir de um determinado tamanho as firmas reduzem a ETR, alinhando-se assim a hipótese do poder político. 
A alavancagem é um determinante da ETR que está relacionada aos benefícios fiscais da dívida. A utilização de dívidas e a minimização de impostos seriam atividades complementares e não substitutas (REGO, 2003, LIN; TONG; TUCKER, 2014). Santoset. al., (2013) explicam que as firmas maiores assumem endividamento elevado por estarem submetidas a uma maior tributação, sendo que esta decisão não seria condizente com a hipótese dos custos políticos, pois tal efetividade implicaria em menores impostos. Poder-se-ia argumentar a favor da hipótese do poder político, mas o motivo para tal decisão não decorre da influência política, explicam os autores.

Quanto àrentabilidade, os estudos sugerem que as empresas mais rentáveis possuem maiores ETRs(GUPTA; NEWBERRY, 1997; PLESKO, 2003; RICHARDSON; LANIS, 2007; ARMSTRONG et. al.,2012).A argumentação teórica é que estasempresas pagariam mais impostos por possuírem lucros mais elevados e, consequentemente,estariam sujeitas aapresentarem ETRs maiores. Outro argumento é que empresas rentáveis seriam eficientes na utilização dos recursos financeiros, permitindo maior investimento em planejamento tributário para gerar ETRs mais baixas.

Em relação a intensidade do capital, a principal decisão está relacionada à possibilidade de dedução dos encargos fiscais provenientes da depreciação dos ativos fixos. Uma vez que a vida útil desses ativos geralmente supera o período de depreciação, empresas mais intensivaspodem gerenciar seus impostosde forma mais flexível através das despesas de depreciação(PLESKO, 2003; LIU; CAO, 2007; CHEN et. al., 2010). Por sua vez, a decisão de investimento em estoques pode ser uma alternativa aos investimentos em ativos fixos (GUPTA; NEWBERRY, 1997). Assim, quanto maior o investimento em capital, menor seria o mesmo em estoque, de modo que quando a empresa investe em estoque está abrindo mão das deduções fiscais provenientes da depreciação dos ativos fixos, ocasionando em maior tributação efetiva. (RICHARDSON; LANIS, 2007; FERNÁNDEZ-RODRÍGUEZ; MARTÍNEZ-ARIAS, 2014; PARISI, 2016).

Diante do exposto, a hipótese de pesquisa assumida é a seguinte:

H1: As empresas brasileiras de capital fechado são propensas a apresentaram Effective Tax Rate (ETR) mais agressivas do que as de capital aberto.

\section{METODOLOGIA}

Esta sessão descreve o método de pesquisa, incluindo a coleta e amostra, tratamento e mensuração das variáveis e os modelos econométricos.

\subsection{Coleta e amostra}

A coleta dos dados deu-se através da base Economática (empresas abertas, listadas na BM\&FBovespa) e da Quantum Axis ${ }^{\circledR}$ (empresas fechadas) e se refere ao reporte anual das demonstrações financeiras nos anos de 2010 a 2015. O recorte entre 2010 e 2015 foi limitado pelas informações da Demonstrações do Fluxo de Caixa (somente a partir de 2010) e pela disponibilidade de dados das empresas fechadas (última informação disponível era de 2015). Da base de dados Quantum Axis ${ }^{\circledR}$ foram coletadas inicialmente as 500 maiores empresas fechadas, 
distribuídas por setores econômicos e, posteriormente foram mantidas na amostra final as empresas de capital fechado, consideradas potenciais participantes do mercado de ações por apresentarem as características necessárias para abertura do seu capital. Através deste critério de coleta é razoável assumir que as empresas que compõem a amostra estão sob o mesmo conjunto de regras fiscais, dado que os impostos sobre o lucro seriam calculados sob a mesma base contábil e tributária, mantendo-as constantes para efeito de análise e garantindo a alíquota nominal máxima de $34 \%$ (importante para efeito de comparação com a alíquota efetiva sobre o lucro). A amostra, incluindo os dois grupos de empresas, compreende 668 empresas com 4.008 observações iniciais, ajustadas posteriormente após tratamento estatístico. A Tabela 1 apresenta a composição da amostra inicial das empresas utilizadas neste trabalho.

Tabela 1. Composição da amostra contemplando as mesmas empresas nos anos de 2010 a 2015

\begin{tabular}{l|c} 
Empresas fechadas & $\mathbf{5 0 0}$ \\
(-) Sociedades de economia mista, sociedade empresária limitada, arrendamento & $(173)$ \\
mercantil, bancos, administradoras (holdings), intermediação financeira e seguradoras e & 327 \\
corretoras. & 373 \\
Total de empresas fechadas após exclusões & $(32)$ \\
Empresas abertas & \\
(-) Bancos, administradoras (holdings), intermediação financeira e seguradoras e & 341 \\
corretoras. & 668 \\
Total de empresas abertas após exclusões & \\
Total de empresas da amostra & \\
\hline
\end{tabular}

Fonte: Quantum Axis ${ }^{\circledR}$ (empresas de capital fechado) e Economática ${ }^{\circledR}$ (empresas de capital aberto).

O período contemplado na pesquisa iniciou-se após a implantação das leis n ⒒638/07 e no 11.941/07 e do Regime Tributário de Transição (RTT) garantindo consistência na contabilidade das empresas investigadas. Contudo, incluiu-se nos modelos de regressão em painel de dados dummies de ano para controlar possíveis influências dos ajustes contábeis feitos pelo Comitê de Políticas Contábeis (CPC) nos anos iniciais, e dos efeitos da crise econômica nos anos finais da amostra.

\subsection{Tratamento e mensuração das variáveis}

A variável dependenteé a Effective Tax Rate (ETR) e a variávelprincipal de análiseé uma dummy(FIRMA) que representa as empresasabertas ou fechadas. Uma vez que não foi possível obter os valores separados dos impostos correntes e diferidos, calculou-se a ETR total (considerada adequada para os propósitos desta pesquisa). Como robustez, foram introduzidas variáveis de controle(Tabela 7) para testar se as mesmas exercem influência na variável principal.Tratou-sea variável ETR com base nos seguintes critérios:( $i$ ) quando o imposto de rendafoi negativo, substitui-se o valor por zero no numerador; (ii) quando o LAIR foi menor que zero no denominador e o imposto de renda foi positivo, colocou-se 1 (um) no numerador. Além 
disso, as ETRs maiores que 1 (um) foramcensuradas por apresentarem condições atípicas.Este tratamento proporcionou modificações em 196 observações de empresas de capital fechado e 23 observações de empresas de capital aberto.

Seguindo a metodologia aplicada por Stickney e McGee (1982), Gupta e Newberry (1997),Richardson eLanis(2007), Kraft(2014) e Ribeiro, Cerqueira e Brandão(2015), o tratamento dado a ETRpermitiu uma redução de perdas da amostra e, consequentemente, ummelhor ajustamento dos modelos.As variáveis contínuas foram padronizadas pelo ativo total e os outliers winsorizados entre1\% e 99\%para amenizar possíveisdistorçõesna interpretação dos resultados.A Tabela 2 apresenta a descrição das variáveis, a forma como foram mensuradas e os trabalhos que as fundamentam.

Tabela2. Especificação das variáveis utilizadas na pesquisa, mensuração e fundamentação

\begin{tabular}{|c|c|c|c|}
\hline Descrição & Variável & Mensuração & Fundamentação \\
\hline Effective Tax Rate & ETR & $\begin{array}{c}\text { Despesa com impostos (IRPJ + } \\
\text { CSLL) / Lucro Antes do Imposto } \\
\text { de Renda - LAIR }\end{array}$ & $\begin{array}{c}\text { Hanlon e Heitzman (2010); Chen } \\
\text { et. al., (2010) }\end{array}$ \\
\hline Status daempresa & FIRMA & $\begin{array}{c}\text { Dummy,sendo } 1 \text { para as } \\
\text { empresas de capital fechado e } \\
0 \text { para as demais }\end{array}$ & $\begin{array}{c}\text { Cloyd, Pratt e Stock, (1996); } \\
\text { Jacob, Rohlfing-Bastian e } \\
\text { Sandner, (2014); Pierk (2016) }\end{array}$ \\
\hline Tamanho da Firma & TAM & Log do ativo total & $\begin{array}{c}\text { Zimmerman (1983); Richardson e } \\
\text { Lanis (2007); Santos, Cavalcante } \\
\text { e Rodrigues (2013) }\end{array}$ \\
\hline Alavancagem & ALAV & $\begin{array}{c}\text { Dívidas de longo prazo / Ativo } \\
\text { total }\end{array}$ & $\begin{array}{c}\text { Rego (2003); Taylor, Richardson } \\
\text { (2014), Santos, Cavalcante e } \\
\text { Rodrigues (2013) }\end{array}$ \\
\hline Rentabilidade & ROA & $\begin{array}{c}\text { Lucro antes do IR e CSLL / Ativo } \\
\text { total }\end{array}$ & $\begin{array}{c}\text { Gupta e Newberry (1997); } \\
\text { Richardson; Lanis (2007); Desai e } \\
\text { Dharmapala (2009) }\end{array}$ \\
\hline Intensidade do Capital & INTCAP & Imobilizado / Ativo total & $\begin{array}{c}\text { Gupta e Newberry (1997); Desai } \\
\text { e Dharmapala (2009) }\end{array}$ \\
\hline Intensidade do Estoque & INTINV & Estoque / Ativo total & $\begin{array}{c}\text { Parisi (2016), Fernández- } \\
\text { Rodríguez e Martínez-Arias } \\
\text { (2014) }\end{array}$ \\
\hline $\begin{array}{l}\text { Estágio do Ciclo de Vida } \\
\text { organizacional - ECV }\end{array}$ & $\begin{array}{l}\text { NASC, CRESC, } \\
\text { MATUR } \\
\text { TURB, DECL }\end{array}$ & $\begin{array}{c}\text { Dummy,sendo } 1 \text { para } \\
\text { determinado ECV e } 0 \text { para os } \\
\text { demais }\end{array}$ & $\begin{array}{l}\text { Dickinson (2011), Hasan et. al., } \\
\text { (2016), Drake, } 2015\end{array}$ \\
\hline Setores econômicos & $\begin{array}{l}\text { SETOR1, SETOR2, } \\
\text { SETOR3, SETOR4, } \\
\text { SETOR5, SETOR6 } \\
\end{array}$ & $\begin{array}{c}\text { Dummy, sendo } 1 \text { para } \\
\text { determinado setor e } 0 \text { para os } \\
\text { demais }\end{array}$ & $\begin{array}{c}\text { Adaptado de Santos, Cavalcante } \\
\text { e Rodrigues (2013) }\end{array}$ \\
\hline Ano & $\begin{array}{c}2010,2011 \\
2012,2013,2014 \\
2015\end{array}$ & $\begin{array}{c}\text { Dummy, sendo } 1 \text { para } \\
\text { determinado ano e } 0 \text { para os } \\
\text { demais }\end{array}$ & - \\
\hline
\end{tabular}

Fonte: Quantum Axis ${ }^{\circledR}$ e Economática ${ }^{\circledR}$.

Observações: IRPJ = Imposto de Renda Pessoa Jurídica. CSLL = Contribuição Social sobre o Lucro Líquido. OsECVs foram classificados segundo o modelo de Dickinson (2011) contemplando os estágios de nascimento, crescimento, 
maturidade, turbulência e declínio. Os setores econômicos foram classificados de 1 a 6 , contemplando, respectivamente, o setor industrial (1), comércio (2), construção civil (3), comunicação, água e energia (4), transporte e serviços relacionados (5)e, outros setores (6).

\subsection{Modelos econométricos}

Inicialmente, foi estimado um modelo de regressão por OLS (OrdinaryLeast Square),por ano, para analisar a associação existente entre as características individuais das empresas brasileiras e a Effective Tax Rate (ETR) - Modelo A. A análise permitirá verificar se há variação no comportamento da variável dependente (ETR) perante a variável de interesse (FIRMA) em cada período. Todos os pressupostos da regressão foram testados e o modelo foi estimado com erros padrão robustos (Tabela 6).

Em seguida foram estimados os modelos de dados em painel.A vantagem de usar dados em painel em relação a dados de corte deve-se ao: (i) controle da heterogeneidade individual, que possibilita medir separadamente os efeitos gerados por conta de diferenças individuais em cada cross-section, além da possibilidade de avaliar a evolução das variáveis de dado indivíduo ao longo do tempo; (ii) aumentoda quantidade de informações, garantindo uma maior variabilidade dos dados, aumento do número de graus de liberdade e maior eficiência na estimação.

Com base nos Testes de Wooldridge e de Wald rejeitaram-se as hipóteses nulas de ausência de autocorrelação e de heteroscedasticidade, respectivamente. A correção da heterocedasticidade deu-se por estimações com erros padrões robustos nas regressões. Ostestes Breusch-Pagan-Godfrey e o teste robusto deHausman foram usadospara a escolha dos modelos de painel. Dado que a variável de interesse (FIRMA) e os setores econômicos (SETOR) são dummies invariantes no tempo, os modelos de efeitos fixos (FE) foram estimados pela abordagem da variável dummy de mínimos quadrados (LSDV - Least Square Dummy Variables). A estimação por LSDV permite que as variáveis constantes no tempo sejam estimadas e seja considerada a heterogeneidade entre as empresas, permitindo que cada uma tenha seu próprio intercepto $\beta_{0 i}$ (GUJARATI; PORTER, 2011).

O pressuposto de normalidade foi relaxado, uma vez que a amostra é relativamente grande havendo uma tendência para a distribuição normal de acordo com o teorema do limite central (GUJARATI; PORTER, 2011). Uma vez que o estudo usa duas bases de dados distintas buscou-se verificar se as médias apresentam diferenças significativas lançando mão dos testes de médias. $O$ teste não paramétrico de Mann-Whitney foi aplicado em conjunto com o Test $t$ por causa do relaxamento do pressuposto de normalidade e para dar mais robustez aos resultados (FÁVERO et. al., 2014).Para determinar a forma mais adequada de rodar o Test $t$ foi verificado em cada ano a igualdade de variâncias pelo Test $F$.Os testes de diferenças de médias levaram à rejeição da hipótese nula de que as médias são diferentes para as ETRs em todos os anos.

A Equação 1 apresenta o modelo final (modelo E da regressão em dados em painel) que testa a influência das características financeiras e operacionais específicas das empresas 
brasileiras de capital fechado e de capital aberto na ETR com inclusão de todas as variáveis explicativas.

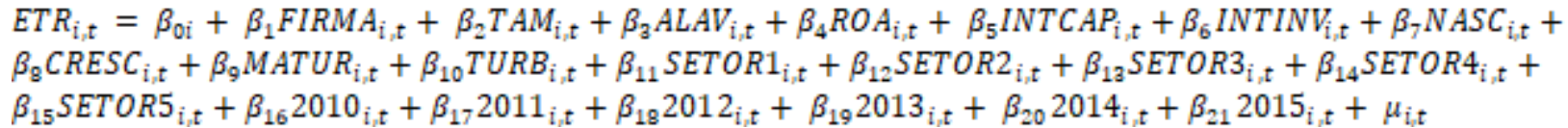

Onde, $E T R_{i, t}=$ Effective Tax Rate (ETR) da firma $i$ no tempo $t$ (exercícios financeiros encerrados em 2010 a 2015). $\beta_{0 i}=$ intercepto invariante no tempo, ou seja, efeitos fixos não observados específicos da firma i. $\beta_{1}$ a $\beta_{21}=$ coeficientes dos determinantes da ETR (Tabela 2 ).

\section{ANÁLISE E DISCUSSÃO DOS RESULTADOS}

Esta sessão apresenta a análise e discussão dos resultados, abrangendo a estatística descritiva e a análise de regressão.

\subsection{Estatística Descritiva}

A Tabela 3 demonstra aestatística descritiva das variáveis contínuas. Percebe-se que as empresas brasileiras apresentam uma ETRmédia de $21,37 \%$ no período, inferior a alíquota efetiva nominal máxima de 34\%.Os valores mínimos e máximos dessa variável são reflexos dos critérios aplicados no tratamento da ETR. A rentabilidade (ROA) apresenta-se com média baixa $(-0,0597)$, indicando que as empresas no período analisado tiveram pouca lucratividade. Observa-se que as empresas são mais intensivas no capital $(36,61 \%)$ em relação aos estoques (5,92\%), sugerindo que os investimentos em imobilizado são priorizados em relaçãoaos estoques.Percebe-se, também, uma grande variação na alavancagem das empresas, uma vez que o desvio padrão é alto em relação a média e há uma distância razoável entre os valores mínimos e máximos desta variável.

Tabela 3. Estatística descritiva das variáveis contínuas. 2010 a 2015

\begin{tabular}{lcccccc}
\hline \multicolumn{1}{c}{ Variáveis } & Obs & Média & Desviopadrão & Mín & P50 & Máx \\
\hline ETR & 3.586 & 0,2137 & 0,3327 & 0 & 0 & 1 \\
Tamanho & 3.585 & 5,8434 & 1,0718 & 1,2041 & 5,9405 & 8,088 \\
Alavancagem & 3.525 & 0,3976 & 0,8807 & 0 & 0,2617 & 11,25 \\
ROA & 3.525 & $-0,0597$ & 0,7489 & $-9,7181$ & 0,0457 & 0,7448 \\
Intensidade do capital & 3.521 & 0,3661 & 0,3052 & 0 & 0,3079 & 0,9903 \\
Intensidade do estoque & 3.525 & 0,0592 & 0,0890 & 0 & 0,0062 & 0,4102 \\
\hline
\end{tabular}

Fonte: Quantum Axis $^{\circledR}$ e Economática ${ }^{\circledR}$.

A Tabela 4 reporta os resultados da estatística descritiva para a variável dependente (ETR) por tipo de empresase por setores econômicos e anos.A divisão da amostra por setores visademonstrar possíveis diferenças nas ETRs provenientes de benefícios fiscais concedidos a 
setores específicos.De forma geral, as ETRs das empresas de capital fechado continuam inferiores às de capital aberto dentro dos setores econômicos, sendo que a menor diferença média é a do setor industrial.

Em relação as empresas de capital aberto, o setor com o menor desvio padrão em relação a média é o decomunicação, agua e esgoto, indicando um comportamento mais homogêneo da ETR, contudo o setor comercial e o de transporte e serviços relacionados apresentam desvio padrão abaixo da média. Este último setor também apresentou as ETRs mais próximas da taxa nominal para ambas as empresas. O setor mais regulamentado, que é o de comunicação, água e energia apresentam uma ETR média de $29,89 \%$. Por sua vez, as empresas fechadas deste setor mantêm um comportamento igual aos outros setores, com ETRs mais agressivas e desvio padrão elevado.

Tabela 4. Estatística descritiva da ETR por empresas abertas e fechadas e setores econômicos. 2010 a 2015

\begin{tabular}{|c|c|c|c|c|c|c|}
\hline \multirow{2}{*}{ Empresas } & $\mathrm{N}$ & Média & SD & Min & P50 & Max \\
\hline & \multicolumn{6}{|c|}{ Setor industrial } \\
\hline Aberta & 615 & 0,2475 & 0,3211 & 0 & 0,1527 & 1 \\
\hline \multirow[t]{2}{*}{ Fechada } & 163 & 0,2314 & 0,4213 & 0 & 0 & 1 \\
\hline & \multicolumn{6}{|c|}{ Setor comercial } \\
\hline Aberta & 115 & 0,2881 & 0,2485 & 0 & 0,2836 & 1 \\
\hline \multirow[t]{2}{*}{ Fechada } & 109 & 0,2037 & 0,3943 & 0 & 0 & 1 \\
\hline & \multicolumn{6}{|c|}{ Setor de construção civil } \\
\hline Aberta & 128 & 0,3298 & 0,3622 & 0 & 0,1622 & 1 \\
\hline \multirow[t]{2}{*}{ Fechada } & 360 & 0,1134 & 0,3076 & 0 & 0 & 1 \\
\hline & \multicolumn{6}{|c|}{ Setor de comunicação, água e esgoto } \\
\hline Aberta & 329 & 0,2989 & 0,3166 & 0 & 0,2322 & 1 \\
\hline \multirow[t]{2}{*}{ Fechada } & 334 & 0,1505 & 0,3501 & 0 & 0 & 1 \\
\hline & \multicolumn{6}{|c|}{ Setor de transporte e serviços relacionados } \\
\hline Aberta & 107 & 0,3429 & 0,2488 & 0 & 0,3375 & 1 \\
\hline \multirow[t]{2}{*}{ Fechada } & 116 & 0,2536 & 0,4287 & 0 & 0 & 1 \\
\hline & \multicolumn{6}{|c|}{ Outros setores } \\
\hline Aberta & 639 & 0,2560 & 0,3027 & 0 & 0,2119 & 1 \\
\hline Fechada & 571 & 0,1048 & 0,2962 & 0 & 0 & 1 \\
\hline
\end{tabular}

Fonte: Quantum Axis ${ }^{\circledR}$ e Economática ${ }^{\circledR}$.

Observações: $\mathrm{N}$ = número de observações. SD = Desvio padrão.

A Tabela 5 apresenta a estatística descritiva da ETR por estágios do ciclo de vida (ECV). Pode-se observar que as empresas de capital aberto possuem mais agressividade na taxa efetiva sobre o lucro no estágio de declínio $(22,4 \%)$, mas em todos os estágios a ETR permanece abaixo da taxa nominal. Observa-se também que o desvio padrão varia menos nos estágios de crescimento e maturidade, demonstrando menor dispersão dos dados nesses estágios. Em relação as empresas de capital fechado, chama a atenção a média de ETR no estágio de 


\section{(9REUNIR}

maturidade de apenas 7,8\%. Em parte, este resultado é reflexo dos ajustes efetuados na ETR (0 1), contudo demonstra a existência de muitas ETR com valores negativos ou próximos de 0 (zero). O desvio padrão elevado e a mediana não capturada indicam a dispersão dos dados dessas empresas no estágio de maturidade. Por sua vez, no estágio de nascimento observa-se a maior média da ETR (30,7\%), mas os dados continuam dispersos e sem capturar a mediana neste grupo de empresas.

Tabela 5. Estatística descritiva da ETR por tipo de empresas e ECV. 2010 a 2015

\begin{tabular}{lccccccc}
\hline \multirow{2}{*}{ Empresas } & & N & Média & SD & Min & P50 & Max \\
\cline { 2 - 8 } ECV NASC & A & 121 & 0,294 & 0,447 & 0,000 & 0,000 & 1,000 \\
& F & 244 & 0,307 & 0,454 & 0,000 & 0,000 & 1,000 \\
\hline \multirow{2}{*}{ ECV CRESC } & $\mathrm{A}$ & 616 & 0,290 & 0,281 & 0,000 & 0,251 & 1,000 \\
& $\mathrm{~F}$ & 372 & 0,164 & 0,361 & 0,000 & 0,000 & 1,000 \\
\hline \multirow{2}{*}{ ECV MATUR } & $\mathrm{A}$ & 712 & 0,269 & 0,254 & 0,000 & 0,245 & 1,000 \\
& $\mathrm{~F}$ & 685 & 0,078 & 0,258 & 0,000 & 0,000 & 1,000 \\
\hline \multirow{2}{*}{ ECV TURB } & $\mathrm{A}$ & 274 & 0,282 & 0,360 & 0,000 & 0,133 & 1,000 \\
& $\mathrm{~F}$ & 260 & 0,110 & 0,306 & 0,000 & 0,000 & 1,000 \\
\hline \multirow{2}{*}{ ECV DECL } & $\mathrm{A}$ & 216 & 0,224 & 0,403 & 0,000 & 0,000 & 1,000 \\
& $\mathrm{~F}$ & 94 & 0,253 & 0,433 & 0,000 & 0,000 & 1,000 \\
\hline
\end{tabular}

Fonte: Quantum Axis ${ }^{\circledR}$ e Economática ${ }^{\circledR}$.

Observações: $\mathrm{N}=$ número de observações. $\mathrm{SD}=$ desvio padrão. $\mathrm{P} 50$ = mediana. $\mathrm{ECV}=$ Estágios do Ciclo de Vida de acordo com o modelo de Dickinson (2011).

\subsection{Análise de Regressão[}

A Tabela 6 apresenta os resultados da análise de regressão estimada por OLS para os anos de 2010 a 2015. Esta é uma análise preliminar sem levar em consideração os controles não observáveis ao longo do tempo. Constatou-se que a variável de interesse (FIRMA) apresentouuma associação negativa com a ETR, com pouca variação entre os anos (de $9 \%$ a $14 \%)$.Desse modo, pode-se avaliar que as empresas de capital fechado são mais propensas a apresentarem ETRs menores.

Destaca-se que no ano de 2015, o ECV-Maturidade apresentou forte relação com a ETR (negativa e significante com $\mathrm{p}<0,001$ ) sugerindo que as empresas neste estágio do ciclo de vida apresentaram ETRs mais agressivas, talvez por efeito da crise econômico e financeira instalada no país naquele período.Quanto aos setores econômicos, destaca-se o setor de transporte e serviços que apresentou uma relação significante e positiva com a ETR nos anos de 2011, 2014 e 
2015. O tamanho da firma também se mostrou associado com a ETR, mas de forma positiva. As demais variáveis não apresentaram significância padronizada no período.

Tabela 6. Resultado das regressões OLS para os determinantes da ETR. 2010 a 2015

\begin{tabular}{|c|c|c|c|c|c|c|}
\hline \multicolumn{7}{|c|}{ Modelo A - OLS } \\
\hline Variáveis & 2010 & 2011 & 2012 & 2013 & 2014 & 2015 \\
\hline Firma & $-0,1039 * *$ & $-0,0989 * * *$ & $-0,1239 * * *$ & $-0,1092 * * *$ & $-0,1309 * * *$ & $-0,1467 * * *$ \\
\hline Tamanho & $0,0335^{*}$ & $0,0477^{* * *}$ & $0,0582 * * *$ & $0,0584^{* * *}$ & $0,0405^{* *}$ & $0,0467^{* *}$ \\
\hline Alavancagem & $-0,0045$ & $-0,0079$ & $-0,0227$ & $-0,0134$ & 0,0027 & $-0,0278$ \\
\hline ROA & $-0,0098$ & $-0,0193$ & $-0,0525$ & $-0,0202$ & $-0,0512$ & 0,0000 \\
\hline Intensidade do capital & 0,0319 & 0,0062 & 0,0004 & 0,0131 & 0,0612 & 0,0839 \\
\hline Intensidade do estoque & 0,0063 & 0,1523 & $0,5028 * *$ & $0,4459 *$ & 0,1626 & 0,3009 \\
\hline Setor 1 - Industria & 0,0063 & 0,0146 & $-0,0573$ & $-0,0505$ & $-0,0213$ & $-0,0069$ \\
\hline Setor 2 - Comércio & $-0,0917^{*}$ & 0,0789 & $-0,0722$ & $-0,0883$ & 0,0779 & 0,1252 \\
\hline Setor 3 - Construção civil & $-0,0735$ & $-0,0124$ & $-0,0879 *$ & $-0,0758$ & $0,1170^{*}$ & $-0,0675$ \\
\hline Setor 4-Com., água e esgoto & 0,0779 & 0,0534 & 0,0387 & $-0,0309$ & $0,0849 *$ & $-0,0092$ \\
\hline Setor 5-Transp. e serv. relac. & 0,1006 & $0,1494 *$ & 0,0556 & 0,0323 & $0,1227^{*}$ & $0,1927 * *$ \\
\hline ECV - Nascimento & 0,0703 & 0,0172 & 0,0975 & 0,0946 & 0,1487 & $-0,1721$ \\
\hline ECV - Crescimento & $-0,0073$ & $-0,0222$ & $-0,0590$ & $-0,0366$ & $-0,0218$ & $-0,2201^{*}$ \\
\hline ECV - Maturidade & $-0,0934$ & $-0,0975$ & $-0,0572$ & $-0,0957$ & $-0,0463$ & $-0,2979 * * *$ \\
\hline ECV - Turbulência & $-0,0106$ & $-0,0841$ & $-0,0087$ & $-0,0560$ & $-0,0242$ & $-0,1520$ \\
\hline Constante & 0,0683 & $-0,0228$ & $-0,0550$ & $-0,0350$ & $-0,0243$ & 0,2178 \\
\hline Observações & 565 & 607 & 617 & 621 & 621 & 490 \\
\hline $\mathrm{R} 2$ & 0,0954 & 0,0108 & 0,1168 & 0,1022 & 0,0927 & 0,1181 \\
\hline
\end{tabular}

Fonte: Quantum Axis ${ }^{\circledR}$ e Economática ${ }^{\circledR}$.

Observações: ${ }^{*} \mathrm{p}<0,05 ;{ }^{* *} \mathrm{p}<0,01 ;{ }^{* * *} \mathrm{p}<0,001$. Todos os pressupostos da regressão foram testados e o modelo foi estimado com erros padrão robustos.

Em seguida, estimou-se um modelo de regressão em dados em painel, cujos resultados estão na Tabela 7. A inclusão gradativa das variáveis visa dar robustez à relação investigada.Destaca-se que os modelos mais complexos (D e E) apresentaram maior poder explicativo (R2 de 47\%).

Os resultados evidenciam uma relação significativa da variável FIRMA com a ETR, indicando que as empresas de capital fechado são mais propensas a apresentaremETRs menores.Esta relação se mantémnos quatro modelos, mas as magnitudes dos coeficientes aumentam de acordo comsua complexidade. $O$ modelo $B$, que não contempla todas as variáveis 
de controle reportou um coeficiente de $-0,1137$, enquanto no modelo $E$, mais completo,o coeficiente foi de $-0,4553$, mostrando que a inclusão dos setores econômicos, dos ECVs e das dummies de ano aumentam o poder explicativo da variável FIRMA. Esses resultados permitem confirmar a hipótese de pesquisa (H1) e está em consonância com Cloydet. al., (1996) e Linet. al., (2013).

Destaca-se que a inclusão dos setores econômicos no modeloC, apesar de ter mantido a relação significativa da variável FIRMA, não melhorou a magnitude dos seus coeficientes. Contudo, observou-se significância estatisticamente negativa para os setores industrial e de comunicação, água e esgoto com à ETR nos modelos D e E, os quais apresentaram maior poder explicativo da variável FIRMA.

Em relação aos ECVs incluídos em conjunto no modelo $D$, percebe-se que o coeficiente da variável FIRMA foi modificado de $-0,1137$ para -0,4423, e os mesmos se mantiveram alto quando controlado por ano (modelo E), sugerindo que os modelos finais capturam de forma mais eficiente a influência que as empresas de capital fechado exercem na ETR. Nota-se, ainda, que os ECVs maturidade e turbulência são significativos $(p<0,05)$, indicando que nesses dois estágios as empresas fechadas são propensas a reduzir a ETR em aproximadamente $6 \%$. Este resultado é parcialmente corroborado por Hasanet. al., (2016) ao confirmar a associação da ETR com os ECVs, mas a direção do sinal é oposta para as empresas maduras.

Quanto as dummies de ano, constatou-se que o ano de 2015 foi o único que mostrou significância estatística e associação positiva. Este resultado pode ter capturado o impacto da crise econômico e financeira que ocorreu nos anos finais dessa amostra, sugerindo que numa situação de crise as empresas são mais propensas a aumentar a ETR. Entretanto, a inclusão dessas dummies não melhorou os coeficientes da variável FIRMA, pois diminuíram de $-0,4423$ para $-0,4253$.

Em relação às demais variáveis de controle, percebeu-se que o tamanho da firma (TAM) se mostrou estatisticamente significante e com coeficientes positivos, sendo que nos modelos $D$ e E apresentaram uma associação ligeiramente mais forte com a ETR (0,0963 e 0,0824). 0 resultado está em consonância com a teoria dos custos políticos, a qual afirma que as empresas maiores aumentam a ETR para evitar ações reguladoras e transferência de riqueza por parte do governo (ZIMMERMAN, 1983; WATTS; ZIMMERMAN, 1986). Por outro lado, esse resultado pode sinalizar para os gestores espaço legal para diminuição da carga tributária de suas empresas no futuro.

A variável ROA também se mostrou significante $(p<0,001)$ nos quatro modelos com os sinais dos coeficientes negativos (aproximadamente 4\%), evidenciando que uma empresa mais lucrativa é capaz de canalizar seus recursos para atividades de tax avoidance, a fim de reduzir sua ETR. Não obstante, este resultado contraria algumas pesquisas que mostraram relação positiva da lucratividade com a ETR (GUPTA; NEWBERRY, 1997; PLESKO, 2003; RICHARDSON; LANIS, 2007; LIU; CAO, 2007; ARMSTRONG; BLOUIN; LARCKER, 2012).

Por fim, em relação a alavancagem, a intensidade do capital e a intensidade do estoque incluídas nos modelos como determinantes da ETR, os resultados não permitiram chegar a conclusões, uma vez que não houve associação estatisticamente significativa dessas variáveis 


\section{(9)REUNIR}

nos modelos estimados. Porém, suas inclusões nos modelos permitiram avaliar melhor a influência da variável principal de interesse (FIRMA) na ETR.

Tabela 7. Resultado das regressões comdados em painel, de efeitos aleatórios (RE) e fixo com LSDV (FE-LSDV), para os determinantes da ETR. 2010 a 2015

\begin{tabular}{|c|c|c|c|c|}
\hline Variáveis & $\begin{array}{c}\text { Modelo B } \\
\text { RE }\end{array}$ & $\begin{array}{c}\text { Modelo C } \\
\text { RE }\end{array}$ & $\begin{array}{l}\text { Modelo D } \\
\text { FE - LSDV }\end{array}$ & $\begin{array}{l}\text { Modelo E } \\
\text { FE - LSDV }\end{array}$ \\
\hline Firma & $-0,1137 * * *$ & $-0,1137^{* * *}$ & $-0,4423 * * *$ & $-0,4253 * * *$ \\
\hline Tamanho & $0,0519 * * *$ & $0,0493 * * *$ & $0,0963 * * *$ & $0,0824 * *$ \\
\hline Alavancagem & $-0,1096$ & $-0,1095$ & $-0,0119$ & $-0,0108$ \\
\hline ROA & $-0,0403^{* * *}$ & $-0,0400 * * *$ & $-0,0497^{* * *}$ & $-0,0438 * * *$ \\
\hline Intensidade do capital & 0,0117 & 0,0157 & $-0,4087$ & $-0,0376$ \\
\hline Intensidade do estoque & 0,0535 & 0,1234 & $-0,4398$ & $-0,4440$ \\
\hline Setor 1 - Industria & - & $-0,0080$ & $-0,5974^{* *}$ & $-0,5669 * *$ \\
\hline Setor 2-Comércio & - & 0,0140 & $-0,3873$ & $-0,3691$ \\
\hline Setor 3 - Construção civil & - & $-0,0174$ & $-0,4008$ & $-0,3909$ \\
\hline Setor 4 - Comunicação, água e esgoto & - & 0,0249 & $-0,5396 *$ & $-0,5632 *$ \\
\hline Setor 5 - Transportes e serv. relacionados & - & $0,1011 *$ & $-0,3652$ & $-0,3433$ \\
\hline ECV - Nascimento & - & - & $-0,0357$ & $-0,0332$ \\
\hline ECV - Crescimento & - & - & $-0,0647$ & $-0,0594$ \\
\hline ECV - Maturidade & - & - & $-0,0686^{*}$ & $-0,0672 *$ \\
\hline ECV - Turbulência & - & - & $-0,0649 *$ & $-0,0675^{*}$ \\
\hline 2011 & - & - & - & $-0,0134$ \\
\hline 2012 & - & - & - & $-0,0103$ \\
\hline 2013 & - & - & - & $-0,0121$ \\
\hline 2014 & - & - & - & $-0,0090$ \\
\hline 2015 & - & - & - & $0,0411^{*}$ \\
\hline Constante & $-0,0427$ & $-0,0403$ & 0,3231 & 0,3841 \\
\hline R2 within & 0,0088 & 0,0079 & - & - \\
\hline R2 between & 0,1101 & 0,1271 & - & - \\
\hline R3 overall & 0,0571 & 0,0641 & - & - \\
\hline $\mathrm{R} 2$ & - & - & 0,4743 & 0,4770 \\
\hline Observações & 3.521 & 3.521 & 3.521 & 3.521 \\
\hline
\end{tabular}

Fonte: Quantum Axis ${ }^{\circledR}$ e Economática ${ }^{\circledR}$.

Observações: $* p<0,05 ;{ }^{* *} p<0,01 ; * * * p<0,001$. O teste robusto de Hausman apontou para dois modelos de efeitos aleatórios ( $A$ e B) e dois modelos de efeitos fixos ( $C$ e D), todos estimados com erros padrão robustos para heterocedasticidade. 


\section{CONSIDERAÇÕES FINAIS}

Este estudo investigou se as empresas brasileiras de capital fechado são propensas a apresentarem Effective Tax Rate (ETR) menores em relação as empresas abertas. Poucos estudos foram nessa direção (CLOYD; PRATT; STOCK, 1996; LIN; MILLS; ZHANG, 2013; JACOB; ROHLFING-BASTIAN; SANDNER, 2014; PIERK, 2016), e no Brasil verificou-se uma lacuna, aqui parcialmente preenchida, uma vez que não foram encontradas pesquisas com este intento.

Os resultados reportados nos modelos de regressão, através das proxies utilizadas, permitem confirmar a hipótese de que as empresas de capital fechado são propensas a apresentarem um maior nível de tax avoidance comparativamente às empresas de capital aberto. Este achado pode estar relacionado à menor pressão do mercado e ao nível de divulgação de informações ao mercado, conforme explicado por Cloyd, Pratt e Stock, (1996) e Lin, Mills e Zhang, (2013). Contudo, sendo um estudo exploratório tal relação pode ser explicada por outros fatores aqui não investigados. É importante destacar que os resultados estão limitados à amostra analisada e aos métodos empregados, não devendo ser generalizados.

Salienta-se que, em todos os modelos, o objetivo principal era estimar a associação entre a variável FIRMA (que representa as empresas abertas ou fechadas) e a ETR, e, em todas as estimações, o coeficiente da FIRMA apresentou sinal negativo, indicando que o fato de a empresa ter seu capital fechado influencia o nível de agressividade da ETR.A intensidade do coeficiente aumentou conforme foram inseridas novas variáveis (setores econômicos, ECV e dummies de ano) nos modelos de painel,mostrando que a inclusão destascontribuíram para confirmar a associação das empresas fechadas com a ETR.Estes resultados estão em consonância com as pesquisas de Cloyd, Pratt e Stock (1996) e Mills e Newberry (2001), mas na contramão de Pierk (2016) que evidenciou ETRs mais agressivas nas empresas abertas.

Como implicações práticas, o estudo contribui para o avanço da pesquisa na área de contabilidade e tributos que trata dos determinantes da Effective Tax Rate, em especial, pela inclusão de empresas de capital fechado na amostra de pesquisa.Ademais, observa-se que, no geral, as empresas brasileiras apresentam ETRs abaixo da taxa nominal de 34\%, mas em todas as análises ficou evidente que as empresas de capital fechado possuem ETRs mais agressivas comparativamente às abertas, conforme hipótese levantada.

É importante enfatizar que a metodologia de cálculo da ETR para análises estatísticas e econométricas não é algo trivial haja vista as diferentes métricas encontradas na literatura. Este artigo seguiu os procedimentos adotados em Gupta e Newberry (1997), Kraft (2014), Ribeiro, Cerqueira e Brandão (2015) e Pierk (2016), embora reconheça que outros métodos, como os usados por Armstrong, Blouin e Larcker, (2012), Santos, Cavalcante e Rodrigues (2013) e Law e Mills (2015) podem gerar diferentes resultados. Acredita-se que somente com o avanço da pesquisa, das informações disponíveis e das discussões acadêmicas possa haver uma maior convergência metodológica acerca da forma mais adequada de mensuração de cálculo da ETR.

Por fim, recomenda-se para pesquisas futuras: $(i)$ o aprofundamento das análises sobre as influências das características individuais das empresas na determinação da ETR, 
considerando aspectos relacionados a governança corporativa, gestão familiar e custos de reputação; e(ii) o uso deoutras medidas tributárias captem outras formas de tax avoidance.

\section{REFERÊNCIAS}

ARMSTRONG, C.S.; BLOUIN, J.L.; LARCKER, D.F. The Incentives for Tax Planning. Journal of Accounting and Economics, vol. 53, 2012, pp. 391-411.

CHEN, S.;CHEN, X.;CHENG, Q.;SHEVLIN. Are family firms more tax aggressive than non-family firms? Journal of Financial Economics, vol. 95, n. 1, 2010,pp. 41-61.

CLOYD, C. B.;PRATT, J.;STOCK, T. The use of financial accounting choice to support aggressive tax positions: Public and private firms. Journal of Accounting research, 1996,pp. 23-43.

DESAI, M. A.;DHARMAPALA, D. Corporate tax avoidance and high-powered incentives. Journal of Financial Economics. Vol. 79, 2006,pp. 145-179.

DICKINSON, V. Cash flow patterns as a proxy for firm life cycle. The Accounting Review, vol. 86, n. 6, 2011,pp. 1969-1994.

DHALIWAL, D. S.;GLEASON, C. A.;MILLS, L. F. Last-chance earnings management: using the tax expense to meet analysts' forecasts. Contemporary Accounting Research, vol. 21, n. 2, 2014, pp. 431-459.

DRAKE, K. (2015). Does firm life cycle explain the relation between book-tax differences and earnings persistence? Working Paper. 2015,Disponívelem: https://oatd.org/oatd/record?record=oai\%5C\%3Aarizonastate\%5C\%3A15021

DYRENG, S.; HANLON, M.;MAYDEW. ETR Long-run corporate tax avoidance. The Accounting Review. Vol. 83, n. 1,2008 , pp. 61-82.

DYRENG, S. D.;HANLON, M.;MAYDEW, E. L. The effects of executives on corporate tax avoidance. The Accounting Review, vol. 85, n. 4, 2010, pp. 1163-1189.

DYRENG, S. D.;HANLON, M.;MAYDEW, E. L.;THORNOCK, J. R. Changes in corporate effective tax rates over the past 25 years. Journal of Financial Economics, vol. 124, n. 3, 2017,pp. 441-463.

FÁVERO, L. P.;BELFIORE, P.;TAKAMATSU, R. T.;SUZART, J. Métodos Quantitativos com Stata: procedimentos, rotinas e análise de resultados. Rio de Janeiro: Elsevier, 2014.

FERNÁNDEZ-RODRÍGUEZ, E.; MARTÍNEZ-ARIAS, A. Determinants of the Effective Tax Rate in the BRIC Countries. Emerging Markets Finance and Trade, vol. 50, 2014, pp. 214-228.

GRAHAM, J. R.;HANLON, M.;SHEVLIN, T.;SHROFF, N. Incentives for tax planning and avoidance: Evidence from the field. The Accounting Review, vol. 89, n. 3, 2014, pp. 991-1023.

GUJARATI, D. M.;PORTER, D. C. EconometriaBásica. 5 ed., Porto Alegre: AMGH, 2011.

GUPTA, S.;NEWBERRY, K.Determinants of the variability in corporate effective tax rates: Evidence from longitudinal data. Journal of Accounting and Public Policy, vol. 16, n. 1, 1997, pp.1-34. 
HANLON, M.;HEITZMAN, S. A review of tax research. Journal of Accounting and Economics, vol. 50, 2010, pp.127-178.

HASAN, M. M.;AL-HADI, A.;TAYLOR, G.;RICHARDSON, G. Does a Firm's Life Cycle Explain Its Propensity to Engage in Corporate Tax Avoidance? European Accounting Review, 2016, pp. 1-33.

HOPE, O-K.;MA, M.;THOMAS, W. B. Tax avoidance and geographic earnings disclosure. Journal of Accounting and Economics, vol. 56, n. 2-3, 2013, pp. 170-189.

JACOB, M.;ROHLFING-BASTIAN, A.;SANDNER, K. Why do not all firms engage in tax avoidance? Facct Center Working Paper, 19, 2014.

KRAFT, A. What really affects German firms' Effective Tax Rate? International Journal of Financial Research, vol. 5, n. 3, 2014, pp. 1-19.

LAW, K.;MILLS, L. F. Taxes and Financial Constraints: Evidence from Linguistic Cues. Journal of Accounting Research, vol. 53, n. 4, 2015, pp. 777-819.

LIN, S.;TONG, N.;TUCKER, A. L. Corporate tax aggression and debt. Journal of Banking \& Finance, vol. 40, 2014, pp. 227-241.

LIN, K. Z.;MILLS, L. F.;ZHANG, F. Public versus private firm responses to the tax rate reduction in China. The Journal of the American Taxation Association, vol. 36, n. 1, 2013, pp. 137-163.

LIU, X.;Cao, S. Determinants of Corporate Effective Tax Rates: Evidence from listed companies in China. The Chinese Economy, vol. 40, 2007, pp. 49-67.

LOPES, T. Custos políticos tributários: o impacto do tamanho na alíquota tributária efetiva. Tese de Doutorado, Universidade de São Paulo, São Paulo, SP, Brasil, 2012. Disponível em: http://http://www.teses.usp.br/teses/disponiveis/12/12136/tde-06022013-190650/en.php

MILLS, L. F.;SANSING, R. C. Strategic Tax and Financial Reporting Decisions: theory and evidence. Contemporary Accounting Research, vol. 17, n. 1, 2000, pp. 85-106.

MILLS, L. F.;NEWBERRY, K. The influence of tax and non-tax costs on book-tax reporting differences: public and private firms. Journal of the American Taxation Association, vol. 23, n. 1, 2001, pp. 1-19.

PARISI, V. The determinants of Italy's corporate tax rates: an empirical investigation. Public and Municipal Finance, vol. 5, n. 1, 2016.

PENNO, M.; SIMON, D. Accounting Choices: Public versus Private Firms. Journal of Business Finance \& Accounting, vol. 13, 1986, pp. 561-569.

PIERK, J. Are Private Firms Really MoreTax Aggressive than Public Firms? WU International Taxation Research Paper Series. Vol 2, 2016.

PLESKO, G. A.An evaluation of alternative measures of corporate tax rates. Journal of Accounting and Economics, vol. 35, n. 2, 2003, pp. 201-226.

REGO, S. O.Tax-Avoidance Activities of U.S. Multinational Corporations. Contemporary Accounting Research, vol. 20, n. 4, 2003, pp. 805-833. 


\section{(O)REUNIR}

RIBEIRO, A. I. M.;CERQUEIRA, A.; BRANDÃO, E. The Determinants of Effective Tax Rates: Firms' Characteristics and Corporate Governance,n. 567, 2015. Universidade do Porto, Faculdade de Economia do Porto.

RICHARDSON, G.;LANIS, R.Determinants of the variability in corporate effective tax rates and tax reform: Evidence from Australia. Journal of Accounting and Public Policy, vol. 26, n. 6, 2007, pp. 689-704.

SANTOS, M. A. C.; CAVALCANTE, P. R. N.;RODRIGUES, R. N. Tamanho da firma e outros determinantes da tributação efetiva sobre o lucro no brasil. Advances in Scientific and Applied Accounting-ASAA,vol. 6, n. 2, 2013, pp. 179-210.

SHACKELFORD, D. A.;SHEVLIN, T.Empirical tax research in accounting. Journal of Accounting and Economics, vol. 31, n. 1-3, 2001, pp. 321-387.

SIEGFRIED, J. The Relationship Between Economic Structure and the Effect of Political Influence: Empirical Evidence from the Federal Corporation Income Tax Program. 1972. Dissertation, Universityof Wisconsin.

SILVA, J. M. A influência do ciclo de vida organizacional sobre o nível de planejamento tributário. 2016. Tese de Doutorado. USP. Ribeirão Preto.

STICKNEY, C. P.;McGEE, V. E. Effective corporate tax rates the effect of size, capital intensity, leverage, and other factors. Journal of accounting and public policy, vol. 1, n. 2, 1982,pp. 125-152.

TAYLOR, G.;RICHARDSON, G.Incentives for corporate tax planning and reporting: Empirical evidence from Australia. Journal of Contemporary Accounting \& Economics,vol. 10, 2014, pp. 1-15.

WATTS, R. L.;ZIMMERMAN, J. L. Positive Accounting Theory. New Jersey: Prentice-Hall, 1986.

ZIMMERMAN, J. L.Taxes and firm size. Journal of Accounting and Economics, Noth-Holland, vol. 5, 1983, pp. 119-149. 